\title{
Impact of a posttraumatic cerebral infarction on outcome in patients with TBI: the Italian multicenter cohort INCEPT study
}

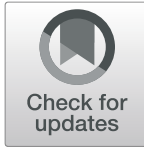

Nicola Latronico ${ }^{1,2+}$, Simone Piva ${ }^{1,2^{*}+}$ (D) Nazzareno Fagoni ${ }^{2,3+}$, Lorenzo Pinelli ${ }^{4}$, Michele Frigerio ${ }^{4}$, Davide Tintori ${ }^{2}$, Maurizio Berardino ${ }^{5}$, Andrea Bottazzi $^{6}$, Livio Carnevale $^{6}$, Tiziana Casalicchio $^{7}$, Carlo Alberto Castioni ${ }^{7}$, Simona Cavallo ${ }^{5}$, Davide Cerasti ${ }^{8}$, Giuseppe Citerio ${ }^{9,10}$, Marco Fontanella ${ }^{11}$, Serena Galiberti ${ }^{12}$, Alan Girardini ${ }^{13}$, Paolo Gritti ${ }^{14}$, Ornella Manara ${ }^{15}$, Paolo Maremmani ${ }^{12}$, Roberta Mazzani ${ }^{16}$, Giuseppe Natalini ${ }^{13}$, Mirko Patassini ${ }^{17}$, Maria Elena Perna ${ }^{18}$, Ilaria Pesaresi ${ }^{19}$, Danila Katia Radolovich ${ }^{6}$, Maurizio Saini ${ }^{10}$, Roberto Stefini ${ }^{20}$, Cosetta Minelli $^{21}$, Roberto Gasparotti ${ }^{1,3}$ and Francesco A. Rasulo ${ }^{1,2+}$

\section{Abstract}

Background: Post-traumatic cerebral infarction (PTCl) is common after traumatic brain injury (TBI). It is unclear what the occurrence of a PTCl is, how it impacts the long-term outcome, and whether it adds incremental prognostic value to established outcome predictors.

Methods: This was a prospective multicenter cohort study of moderate and severe TBI patients. The primary objective was to evaluate if PTCl was an independent risk factor for the 6-month outcome assessed with the Glasgow Outcome Scale (GOS). We also assessed the PTCl occurrence and if it adds incremental value to the International Mission for Prognosis and Clinical Trial design in TBI (IMPACT) core and extended models.

Results: We enrolled 143 patients, of whom 47 (32.9\%) developed a PTCI. In the multiple ordered logistic regression, PTCI was retained in both the core and extended IMPACT models as an independent predictor of the GOS. The predictive performances increased significantly when PTCI was added to the IMPACT core model (AUC $=0.73,95 \%$ C.I. 0.66-0.82; increased to $A \cup C=0.79,95 \% \mathrm{Cl} 0.71-0.83, p=0.0007$ ) and extended model ( $A \cup C=0.74,95 \%$ C.I. $0.65-0.81$ increased to AUC $=0.80,95 \%$ C.I. 0.69-0.85; $p=0.00008$ ). Patients with PTCI showed higher ICU mortality and 6-month mortality, whereas hospital mortality did not differ between the two groups.

Conclusions: PTCl is a common complication in patients suffering from a moderate or severe TBI and is an independent risk factor for long-term disability. The addition of PTCI to the IMPACT core and extended predictive models significantly increased their performance in predicting the GOS.

Trial registration: The present study was registered in ClinicalTrial.gov with the ID number NCT02430324.

Keywords: Traumatic brain injury, Posttraumatic cerebral infarction, Long term outcome, Disability

\footnotetext{
* Correspondence: simone.piva@unibs.it

${ }^{\dagger}$ Nicola Latronico, Simone Piva, Nazzareno Fagoni and Francesco A. Rasulo contributed equally to this work.

'Department of Medical and Surgical Specialties, Radiological Sciences and Public Health, University of Brescia, Brescia, Italy

${ }^{2}$ Department of Anesthesia, Intensive Care and Emergency, ASST Spedali

Civili University Hospital, Piazzale Ospedali Civili 1, 25121 Brescia, Italy

Full list of author information is available at the end of the article
}

(c) The Author(s). 2020 Open Access This article is distributed under the terms of the Creative Commons Attribution 4.0 International License (http://creativecommons.org/licenses/by/4.0/), which permits unrestricted use, distribution, and reproduction in any medium, provided you give appropriate credit to the original author(s) and the source, provide a link to the Creative Commons license, and indicate if changes were made. The Creative Commons Public Domain Dedication waiver (http://creativecommons.org/publicdomain/zero/1.0/) applies to the data made available in this article, unless otherwise stated. 


\section{Background}

A traumatic brain injury (TBI) is a leading cause of mortality and morbidity mostly among young people; although, its incidence is increasing in older people, particularly in high-income countries [1]. The outcome of a TBI depends on several factors, including the patients' characteristics, disease severity at admission, and complications arising during its clinical course. Multivariable prognostic models such as the International Mission for Prognosis and Clinical Trial design in TBI (IMPACT) have shown that most prognostic information is contained in a core set of three predictors: age, the Glasgow Coma Scale (GCS) motor score (GCSm), and pupillary reactivity [2]. IMPACT also provided an extended prognostic model, which adds the brain computed tomography (CT) classification and secondary cerebral insults, such as hypoxia and hypotension, to the core variables. Both core and extended IMPACT models focus on identifying prognostic factors at baseline and do not include predictors from the intensive care unit (ICU) stay [3]. Posttraumatic cerebral infarction (PTCI) is a common complication of a TBI in the acute stage of the disease. A PTCI is frequent in patients who die after a moderate or severe TBI, with a reported incidence in post-mortem studies up to $90 \%$. The antemortem occurrence rate of a PTCI has been assessed in six single-center studies, of which only one was prospective, and the rate varied between $1.9 \%$ and $20.3 \%$ [4-9]. None of these studies investigated whether PTCI adds incremental value to current prognostic models. Therefore, we planned a multicenter, prospective observational cohort study in patients with a moderate or severe TBI to investigate: (1) the impact of PTCI on the 6-month outcome evaluated by the Glasgow Outcome Scale (GOS), (2) if the PTCI adds incremental value beyond that provided by the IMPACT prediction models on the GOS at 6 months, and (3) the occurrence of PTCI in the study population.

\section{Methods}

In the present prospective observational cohort study (ClinicalTrials.gov Identifier: NCT02430324), we included all consecutive adult patients aged $\geq 16$ years with a moderate (post-resuscitation GCS 12 to 9) or severe (post-resuscitation GCS 8 to 3) TBI who were admitted to the ICUs of nine Italian trauma centers from December 2009 to December 2012. Exclusion criteria were a history of cerebral ischemia, CT evidence of brain ischemia at admission, absence of invasive intracranial pressure (ICP) monitoring, and patients with a GCS score of 3 and unreactive pupils.

\section{Patient management}

All patients were intubated, mechanically ventilated, underwent ICP and arterial blood pressure monitoring and ECG and were monitored for peripheral oxygen saturation and end-tidal $\mathrm{CO}_{2}$. Management was in accordance with international recommendations aimed at aggressively treating intracranial hypertension and rapidly correcting secondary cerebral insults [10].

\section{Ethics approval}

The study was conducted in accordance with the Declaration of Helsinki and was approved by the local Ethics Committees of each participating center. Patients' informed consent was waived due to the lack of definition of a legal representative of temporarily incapacitated adult patients in the Italian legislation [11]. The informed consent was obtained from the surviving patients as soon as they regained their mental competency. Family members received detailed information on the study scope and protocol. We followed the STROBE (Strengthening the Reporting of Observational Studies in Epidemiology) guidelines for reporting cohort studies [12].

\section{Data collection}

Data on age, sex, GCS, the ICU and hospital length of stay (LOS), days of mechanical ventilation, type of surgery, major cardio-circulatory events (systemic hypotension, life-threatening cardiac arrhythmias, cardiac arrest), brain CT scans (see below), and GOS were prospectively collected. Several of these variables are risk factors for PTCI development, and their association with PTCI risk in this patient population will be investigated and reported in a separate article.

\section{Imaging}

Posttraumatic brain CT findings were classified according to Marshall et al. $[13,14]$. Brain CT scans were performed at hospital admission and then repeated within $24 \mathrm{~h}$ or within $12 \mathrm{~h}$ if the first CT scan had been obtained within $3 \mathrm{~h}$ after injury, in case of neurological deterioration or an increase in ICP [15-17]. A third CT scan might be scheduled on the 3rd day post-trauma [15]. Follow-up brain CTs were performed at the discretion of the attending physicians of each participating center.

The final diagnosis of PTCI with identification of the time of onset and the type of infarction (territorial cerebral infarction, watershed cerebral infarction, and nonterritorial, non-watershed cerebral infarction, see below), as well as the presence of cerebral herniation, CT signs of intracranial hypertension, Marshall CT score, presence of subarachnoid hemorrhage $(\mathrm{SAH})$ or epidural hematoma $(\mathrm{EDH})$, and the midline shift was performed after central revision of the entire brain CT data set of all patients enrolled. Two senior neuro-radiologists (LP, MF) of the University of Brescia, who were blinded to each other's diagnosis and to the patient's outcome, assessed the brain CT based on DICOM (Digital 
Imaging and Communications in Medicine) scans of each patient in duplicate. Each neuroradiologist independently performed a "one shot" assessment of the entire neuroradiological history of each patient, with an immediate comparison of any questionable findings in a given $\mathrm{CT}$ scan to several previous and following exams, thus making the final diagnosis of cerebral infarction much more reliable. In case of discordance, an agreement was reached by a third neuro-radiologist (RG).

Only cerebral infarction that developed after trauma was considered; ischemic lesions identified on the first CT scan whose density remained unchanged during the neuroradiologic follow-up were considered old infarcts and ignored. Moreover, subtle brain CT hypodensities of uncertain classification due to indistinct margins and no clear mass effect at the first scan were diagnosed as PTCI if one or more of the following findings were present in the follow-up CT exams: (1) an increased hypodensity in the following $24-48 \mathrm{~h}$, with progressive delineation of sharp margins; (2) a wedge-shaped lesion with clear effacement of the cerebral sulci when the lesion was cortical, often in a specific vascular territory of a major cerebral artery; (3) a progressive attenuation of the CT findings in the subacute phase of the ischemia (the so-called "fogging effect"). Conversely, subtle brain CT hypodensities suggesting PTCI in a given brain CT scan, which (1) faded away at the $24 \mathrm{~h}$ CT control, or (2) completely disappeared at longer CT follow-up, or (3) did not follow the expected CT changes for an ischemic lesion, were discarded as a non-ischemic lesion.

Following the definition used in our previous study [4], cerebral infarction was classified according to the following criteria [18-20]: (1) territorial cerebral infarction: well demarcated hypodense lesions within a defined cerebral vascular territory, involving the entire arterial territory (complete infarct) or only part of it (incomplete infarct); the vascular territories considered were the anterior cerebral artery (ACA), middle cerebral artery (MCA), posterior cerebral artery (PCA), lenticulostriate arteries (LSAs), anterior choroidal arteries, thalamo-perforating arteries (TPAs), basilar artery (BA), anterior-inferior cerebellar artery, superior cerebellar artery (SCA), and posterior-inferior cerebellar artery (PICA); (2) watershed cerebral infarction: well demarcated hypodense lesions positioned in boundary zones between the territories of the ACA, MCA, and PCA (superficial or leptomeningeal border zones) or in the terminal zones of the perforating arteries within the deep white matter (deep or medullary border zones); (3) non-territorial, non-watershed cerebral infarction: single or multiple hypodense lesions, unilateral, bilateral, or multifocal with marked borders without a precise localization in a vascular territory.

\section{Outcomes}

The primary outcome was the GOS at 6 months $(5=\operatorname{good}$ recovery; $4=$ moderate disability; $3=$ severe disability; $2=$ vegetative state; $1=$ death) [21]. In particular, we wanted to evaluate if a PTCI was an independent risk factor for the GOS and if it added incremental value in predicting long-term outcome compared with the IMPACT prediction model. The secondary outcomes were (1) the occurrence rate of PTCI in patients with a moderate or severe TBI and (2) the association of PTCI with hospital and ICU mortality, as well as the 6-month mortality. The occurrence rate was also calculated as the number of cases of PTCI during the observation period divided by the total number of patients enrolled in the study [22].

\section{Statistical analysis}

Continuous variables are presented as the mean and standard deviation if normally distributed; while the median and interquartile range was used for non-normally distributed continuous variables and ordinal variables. Discrete variables are reported as the count and percentage. We did not have any missing data at baseline or follow-up (GOS at 6 months). Differences between patients with and without PTCI were tested using the $t$ test or Mann-Whitney $U$ test, as appropriate, for continuous variables (age, ICU LOS, hospital LOS, days of mechanical ventilation), and the $\chi^{2}$ test for binary and categorical variables (secondary cerebral insults, coma, SAH or EDH, Marshall brain CT). We performed a sample size calculation for the association of PTCI with the dichotomized GOS at 6 months (unfavorable outcome, GOS $\leq 3)$. We estimated that 116 patients were needed to obtain a power of $80 \%$ and to detect a minimum odds ratio of 3 , considering a prevalence of unfavorable outcome in patients without PTCI of $25 \%$ at a two-sided significance level of 0.05 .

Ordered logistic regression (proportional odds logistic regression) was used to assess whether PTCI could predict the 6-month GOS (ordinal outcome) [23] using simple regression analysis and whether PTCI remained an independent predictor after adding the predictors from the IMPACT models using multiple regression analysis. In particular, we first assessed the association of the GOS with the variables included in the IMPACT core model (age, GCSm, and pupillary reactivity) and in the extended model (core variables plus Marshall CT classification scale, SAH or EDH, and secondary cerebral insults, in particular, hypoxia and hypotension). We then added PTCI to each model to evaluate its independent association with the GOS [3]. The proportionality assumption was checked for each selected predictor.

To assess the incremental predictive performance of the model when adding PTCI, we dichotomized the 6-month GOS into "unfavorable" (GOS $\leq 3)$ and "favorable" (GOS 4 
and 5) outcomes, performed logistic regression analysis for the core and extended models with and without PTCI, and compared the area under the receiver operating characteristic curve (AUC) of the models with PTCI versus those without PTCI. The AUC varies between 0.5 (a noninformative model) and 1.0 (a perfect predictive model). Differences in the AUC between models were tested using the roc.test function in $\mathrm{R}$ (package pROC). To internally validate our model and to avoid over-optimism, we used a bootstrap procedure [24] using the "auc.adjust" $\mathrm{R}$ function. Finally, we repeated the AUC comparison using the AUC corrected for optimism. All statistical tests were two-tailed, and statistical significance was defined as $p<$ 0.05. All statistical analyses were performed using $R$ (3.0.3).

\section{Results}

During the study period, 487 patients with a TBI were admitted to the 9 participating ICUs with a final 143 patients (29.3\%) enrolled in the study (Fig. 1). Of these, 47 (32.9\%) patients developed a PTCI. There were no differences in patients with and without PTCI in terms of age, severity of the TBI, presence of SAH or EPH on the admission brain CT, hospital LOS, and days of mechanical ventilation (Table 1, Table 2). The incidences of intrahospital hypotension and hypoxia, pupillary light reflex abnormalities, and evacuated mass lesions (defined according to Marshall brain CT classification) were higher in patients developing a PTCI (Table 1). A total of 94 cerebral infarctions developed in 47 patients, 81 were territorial (86.2\%) and 8 were watershed (8.5\%). Five infarctions could not be attributed either to territorial or watershed types. Territorial infarctions were in the area of the MCA $(n=17 ; 18.1 \%)$ ACA, $(n=18 ; 19.1 \%)$, PCA $(n=21 ; 22.3 \%)$, LSA $(n=8 ; 8.5 \%)$, TPA $(n=7 ; 7.4 \%)$, BA $(n=3 ; 3.2 \%)$, SCA $(n=3 ; 32 \%)$, PICA $(n=1 ; 1.1 \%)$, and anterior communicating artery (AcoA) $(n=3 ; 3.2 \%)$. Watershed infarctions were in the boundary zones $(n=$ $3 ; 3.2 \%)$ and terminal zones $(n=5 ; 5.3 \%)$. The mean

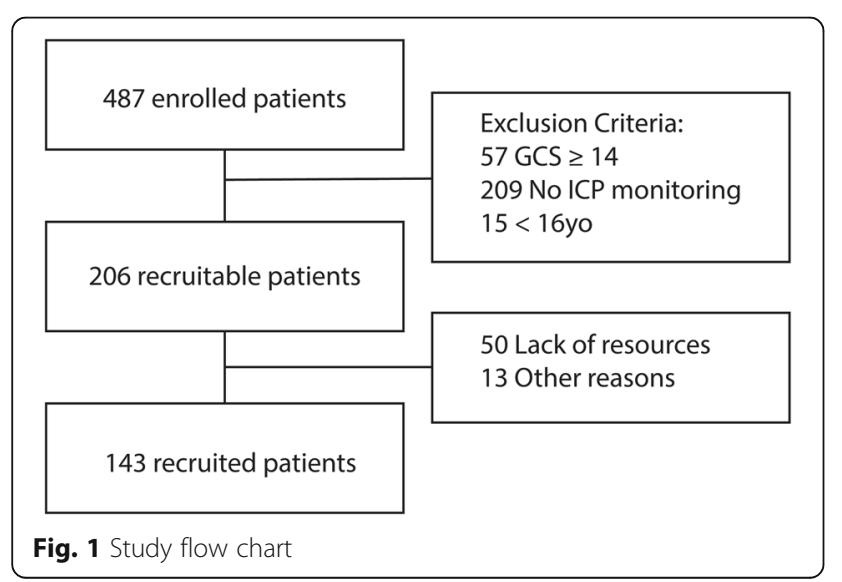

(SD) onset time of a PTCI was 6.2 (11.4) days with an early peak within $24 \mathrm{~h}$ (21 cases, 39.6\%), a late peak between 3 and 7 days (14 cases, 26.4\%), and 4 cases (7.6\%) between 24 and 48 h, Fig. 2 .

After the central revision of the entire brain CT dataset, we correctly identified 10 patients with PTCI, missed at the initial evaluation; conversely, 7 patients initially classified as PTCI were subsequently classified as nonPTCI.

In the simple ordered logistic regression, the GOS was significantly worse in patients with a PTCI than patients without a PTCI, with a higher proportion of patients with a severe disability and death and a lower proportion of patients with good recovery and moderate disability (Table 2). In the multiple ordered logistic regression, a PTCI was retained in both the core and the extended models (Table 3a, b) as an independent predictor of the GOS. The predictive performances of the obtained models (Fig. 3) were good and increased significantly when PTCI was added to the IMPACT core model (AUC $=0.73,95 \%$ C.I. $0.66-0.82$ increased to $\mathrm{AUC}=0.79,95 \%$ CI $0.71-0.83$; 0.0007 ) and the extended model (AUC $=0.74,95 \%$ C.I. $0.65-0.81$ increased to $\mathrm{AUC}=0.80,95 \%$ C.I. 0.69-0.85; $p=0.00008)$. The results were replicated after correcting the AUC for optimism (Fig. 4 and Table 4). Patients with a PTCI showed higher ICU mortality (10 patients [21.3\%] vs one patient $[1.0 \%], p<0.0001)$ as well as higher 6month mortality (13 patients [27.7\%] vs 7 patients [7.3\%], $p<0.0001)$; whereas hospital mortality did not differ between the two groups (Table 2).

\section{Discussion}

In this multicenter prospective cohort study, we found that a PTCI is an independent predictor of an unfavorable 6-month outcome and its addition to the IMPACT core and extended models increased their performance in predicting the GOS. Moreover, we confirmed that PTCI is a frequent complication occurring in more than one-third of patients suffering severe or moderate TBI. Most of the PTCIs were territorial, affecting one or more cerebral artery territories, and developed early during the ICU stay.

This is the first prospective study showing a PTCI has an independent effect on a patient's long-term outcome. Previous studies were either post-mortem neuropathological investigations or ante-mortem retrospective clinical investigations. Among the latter, the GOS was assessed in four single-center studies at 3 months [5] or 6 months $[4,9]$; while in one study, the timing of the GOS was not reported [8]. These studies showed increased morbidity [4], increased mortality [9], increased morbidity and mortality [8], or no difference [5] in patients with a PTCI compared with patients without. In three of these studies $[4,5,9]$, the impact of a PTCI was assessed while 
Table 1 Demographic and clinical data

\begin{tabular}{|c|c|c|c|}
\hline Variables & PTCI $(n=47 ; 32.9 \%)$ & Non-PTCI $(n=96 ; 67.1 \%)$ & $p$ value \\
\hline Age, mean (SD) years & $43.8(17.2)$ & $42.1(18.4)$ & 0.606 \\
\hline Sex, no. (\%) males & $38(80.8 \%)$ & $75(78.1 \%)$ & 0.141 \\
\hline Admission GCS, median (IQR) & $6(4-8)$ & $7(5-9)$ & 0.704 \\
\hline Severe head trauma, no. (\%) & $37(78.7 \%)$ & $70(72.9 \%)$ & 0.452 \\
\hline Moderate head trauma, no. (\%) & $10(21.3 \%)$ & $26(27.1 \%)$ & 0.452 \\
\hline \multicolumn{4}{|c|}{ Secondary cerebral insults in the first $48 \mathrm{~h}, \mathrm{no}$. (\%) } \\
\hline Pre-hospital hypoxia & $5(10.6 \%)$ & $7(7.3 \%)$ & 0.460 \\
\hline Intra-hospital hypoxia & $7(14.9 \%)$ & $13(13.5 \%)$ & 0.048 \\
\hline Cardiac arrest & $11(23.4 \%)$ & $1(1.0 \%)$ & $<0.0001$ \\
\hline Pre-hospital systemic hypotension & $8(17.0 \%)$ & 39 & 0.021 \\
\hline Intra-hospital systemic hypotension & $12(25.5 \%)$ & 35 & 0.274 \\
\hline Hyperthermia intra-H & $13(27.7 \%)$ & 34 & 0.289 \\
\hline \multicolumn{4}{|l|}{ Pupillary light reflex, no. (\%) } \\
\hline Both present & $30(63.8 \%)$ & $85(88.5 \%)$ & 0.002 \\
\hline One present & $7(14.9 \%)$ & $3(3.1 \%)$ & \\
\hline Both absent & $10(21.3 \%)$ & $8(8.3 \%)$ & \\
\hline SAH on admission brain CT, no. (\%) & $33(70.2)$ & $65(67.7)$ & 0.849 \\
\hline EDH on admission brain CT, no. (\%) & $15(31.9)$ & $27(28.1)$ & 0.697 \\
\hline \multicolumn{4}{|c|}{ Admission brain CT, Marshall CT classification, no. (\%) } \\
\hline Diffuse injury I & $1(2.3)$ & $0(0.0)$ & 0.035 \\
\hline Diffuse injury ॥ & $10(23.3)$ & $42(46.7)$ & \\
\hline Diffuse injury III & $3(7.0)$ & $4(4.4)$ & \\
\hline Diffuse injury IV & $2(4.7)$ & $5(5.6)$ & \\
\hline Evacuated mass lesions & $25(58.1)$ & $36(40.0)$ & \\
\hline Non-evacuated mass lesions & $2(4.7)$ & $3(3.3)$ & \\
\hline
\end{tabular}

Abbreviation: GCS Glasgow Coma Scale, SAH traumatic subarachnoid hemorrhage, EDH epidural hematoma, ICU-LOS intensive care unit length of stay, H-LOS hospital length of stay, PTCI posttraumatic cerebral infarction

Table 2 Outcomes data

\begin{tabular}{|c|c|c|c|}
\hline Variables & PTCI $(n=47 ; 32.9 \%)$ & Non-PTCI $(n=96 ; 67.1 \%)$ & $p$ value \\
\hline Days of mechanical ventilation, mean (SD) & $16.0(9.3)$ & $14.3(10.4)$ & 0.339 \\
\hline ICU-LOS, mean (SD) & $21.8(14.5)$ & $19.9(11.7)$ & 0.042 \\
\hline H-LOS, mean (SD) & $43.1(46.2)$ & $45.7(40.6)$ & 0.735 \\
\hline \multicolumn{4}{|l|}{ Primary outcome, 6-month GOS, no. (\%) } \\
\hline Good recovery-5 & $6(13.0 \%)$ & $36(37.9 \%)$ & \multirow[t]{5}{*}{$<0.0001$} \\
\hline Moderate disability—4 & $9(19.6 \%)$ & $32(33.7 \%)$ & \\
\hline Severe disability-3 & $15(32.6 \%)$ & $16(16.8 \%)$ & \\
\hline Vegetative state-2 & $3(6.5 \%)$ & $4(4.2 \%)$ & \\
\hline Death-1 & $13(28.3 \%)$ & $7(7.4 \%)$ & \\
\hline \multicolumn{4}{|l|}{ Secondary outcomes } \\
\hline ICU mortality, no. (\%) & $10(21.3 \%)$ & $1(1.0 \%)$ & $<0.0001$ \\
\hline H mortality, no. (\%) & $12(25.5 \%)$ & $5(5.2 \%)$ & 0.980 \\
\hline 6-month mortality, no. (\%) & $13(27.7 \%)$ & 7 (7.3\%) & $<0.0001$ \\
\hline
\end{tabular}




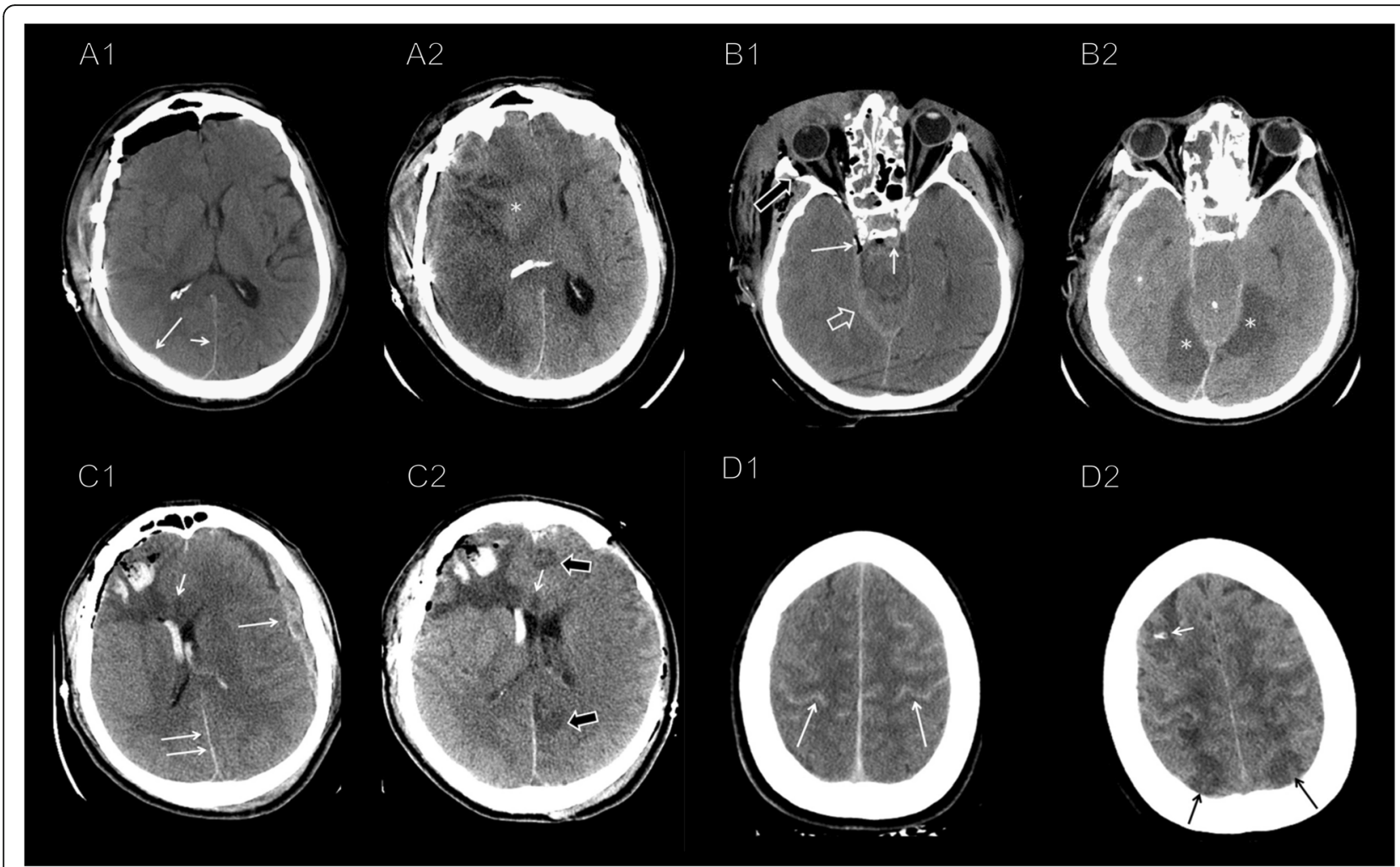

Fig. 2 CT scan showing posttraumatic cerebral infarction (PTCI). A1 MCA PTCl: acute parietal subdural hematoma on the right side (long arrow), extending to the falx (short arrow). A2 CT scan 9 days later showed an acute ischemic lesion in the superficial territory of the right MCA (preserved right lenticular nucleus, white *). B2 PCA PTCl: acute subdural hematoma along the right side of the tentorium (empty arrow), extra-axial blood in the prepontine cistern (short arrow), and small para sellar bubble air (long arrow) on admission brain CT. B2 Brain CT scan at 15 days showed complete effacement of the basal cisterns and bilateral temporo-occipital hypodensities $(*)$, consistent with acute ischemic lesions in the territory of both PCA. C1 ACA PTCl: hemorrhagic contusions of the right frontal lobe mixed with air and perilesional vasogenic edema, intraventricular hemorrhage, a thick left frontoparietal acute subdural hematoma (long arrow) with midline shift to the right, and a thin acute subdural hematoma along the posterior falx (double arrows). C2 Left frontoparietal craniectomy and hematoma evacuation showed multifocal hypodensities in the anterior and posterior portion of the left cingulate gyrus (white outlined arrows), consistent with acute ischemic lesions in the territory of the left ACA. The small hypodensity in the genu of the corpus callosum (short arrow), barely visible in the first exam, is consistent with a shear-strain injury. D1 Superficial watershed PTCl: thick acute subdural hematomas along the whole tentorium and the left frontotemporal convexity. Diffuse subarachnoid hemorrhage is also visible at the vertex (long white arrows). D2 Bilateral cortical hypodensities in the posterior parasagittal regions (black arrows), consistent with acute watershed ischemia at the boundary zone between the MCA and ACA territories. Note the probe for the intracranial pressure monitoring in the left frontal lobe (short white arrow)

considering the role of other predicting variables, such as age and GCS, using multiple regression analysis. However, none of these studies demonstrated that a PTCI adds value to risk prediction models that include validated factors, as we showed here. We added PTCI to the core and extended IMPACT models, which have been extensively validated with various datasets. The IMPACT models focus on baseline prognostic factors and do not include variables that develop throughout the disease process [3]. Therefore, our research expands the results of IMPACT, showing that patients developing a PTCI during the acute stage of disease have a five-fold increased risk for a poor outcome, independently from important factors such as age, motor score, pupillary reactivity, hypotension and hypoxia, brain $\mathrm{CT}$, and the presence of posttraumatic $\mathrm{SAH}$ or EDH.
The AUC increased significantly from 0.73 to 0.79 and from 0.74 to 0.80 when the PTCI was added to the core and extended IMPACT models, respectively; although, these are already strong predictive models. Since the increase in the AUC greatly depends on the strength of the baseline model, the stronger the baseline model, the lesser the expected increase in the AUC [26]. This result further confirms that a PTCI is an important predictor of outcome in patients with a moderate or severe TBI. Our finding that a PTCI is a key independent predictor of long-term morbidity in TBI survivors is highly clinically plausible. Residual morbidity in patients suffering from cerebral infarction is high, with $13 \%$ of survivors discharged to institutional care [27]. Almost half of all elderly people suffering from an ischemic stroke have hemiparesis and cognitive impairment causing moderate 
Table 3 Adjusted ordered logistic regression for the Glasgow Outcome Scale (GOS). Each panel (A and B) includes the IMPACT model with the relative OR on the left (the core model in panel A and the extended model in panel B), and the recalculated OR when PTCl was added as a covariate on the right

\begin{tabular}{|c|c|c|c|c|c|c|}
\hline Panel A & \multicolumn{3}{|c|}{ IMPACT core model } & \multicolumn{3}{|c|}{ IMPACT core model plus PTCI } \\
\hline GOS & OR & 95\% C.I. & $p$ & OR & 95\% C.I. & $p$ \\
\hline Age & 1.04 & $1.02-1.06$ & $<0.0001$ & 1.04 & $1.02-1.06$ & $<0.0001$ \\
\hline GCSm & 0.43 & $0.22-0.84$ & 0.011 & 0.45 & $0.23-0.90$ & 0.025 \\
\hline Pupils & 3.00 & $1.29-7.14$ & 0.048 & 1.91 & $0.79-4.67$ & 0.150 \\
\hline PTCl & - & - & - & 3.88 & $1.85-8.34$ & $<0.0001$ \\
\hline Panel B & \multicolumn{3}{|c|}{ IMPACT extended model } & \multicolumn{3}{|c|}{ IMPACT extended model plus PTCI } \\
\hline GOS & OR & 95\% C.I. & $p$ & OR & 95\% C.I. & $p$ \\
\hline Age & 1.04 & $1.01-1.06$ & $<0.0001$ & 1.04 & $1.02-1.06$ & $<0.0001$ \\
\hline GCSm & 0.43 & $0.21-0.84$ & 0.016 & 0.46 & $0.22-0.91$ & 0.025 \\
\hline Pupils & 3.32 & $1.33-8.51$ & 0.031 & 2.26 & $0.89-5.82$ & 0.085 \\
\hline Hypotension & 1.60 & $0.51-3.78$ & 0.310 & 0.52 & $0.78-4.99$ & 0.147 \\
\hline Hypoxia & 1.41 & $0.26-1.89$ & 0.473 & 1.55 & $0.56-4.23$ & 0.389 \\
\hline SAH & 1.23 & $0.59-2.58$ & 0.555 & 1.17 & $0.56-2.49$ & 0.689 \\
\hline $\mathrm{EDH}$ & 0.93 & $0.44-1.97$ & 0.920 & 0.83 & $0.12-131.36$ & 0.647 \\
\hline Marshall CT score & 2.76 & $0.06-66.33$ & 0.720 & 4.42 & $0.01-8.31$ & 0.385 \\
\hline PTCl & - & - & - & 4.77 & $2.19-10.67$ & $<0.0001$ \\
\hline
\end{tabular}

GCSm Glasgow Coma Scale motor score, OR proportional odds ratio, C.I. confidence interval, SAH traumatic subarachnoid hemorrhage, EDH epidural hematoma. Hypotension and hypoxia refer to both pre-hospital and intra-hospital periods

to severe disability [28]. With severe stroke requiring ICU admission and mechanical ventilation, as many as two-thirds of surviving patients are left with a severe persisting disability [29]. The ICU mortality was significantly higher in patients with a PTCI, suggesting that a PTCI might be a proxy of the severity of the TBI; however, a multivariable analysis could not be performed due to the small number of deaths.
We found that PTCI was more frequent than in our previous retrospective study (32.9\% vs $19.1 \%$ ) [4]. In prospective cohort studies, the selection of patients through application of the inclusion and exclusion criteria is more accurate compared to retrospective studies, as it is the measurement of exposures before the outcome occurs, thereby establishing temporality and outcome. The availability of newer brain CT scanners, with higher sensitivity
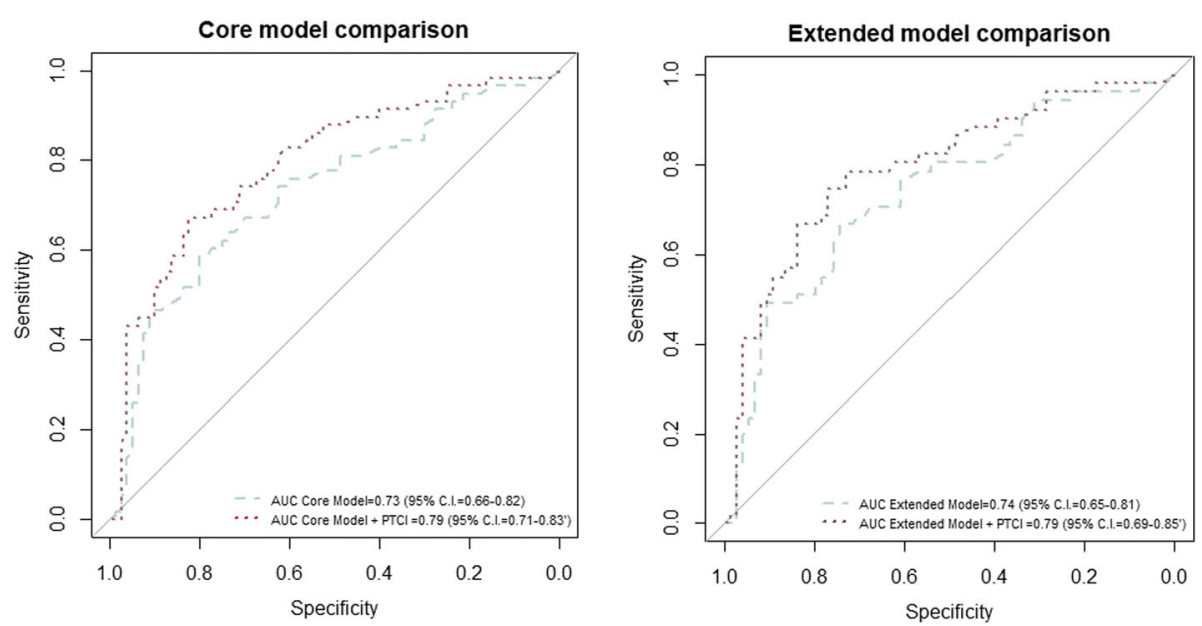

Fig. 3 ROC curves for the core and extended IMPACT models with the addition of PTCI. Comparison of ROC curves and AUCs with and without the addition of PTCl, for both the core and extended models ( $p$ values for the difference in AUC: $p=0.05$ for core model, $p=0.049$ for the extended model). IMPACT: International Mission on Prognosis Analysis of Clinical Trials in Traumatic Brain Injury 


\section{Core Model}
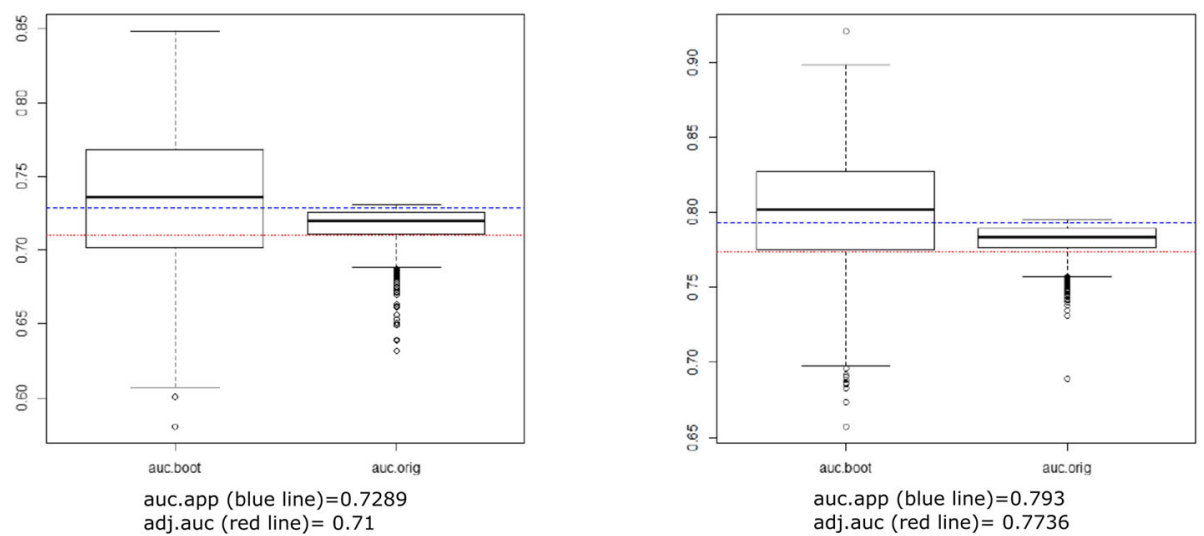

Extended Model
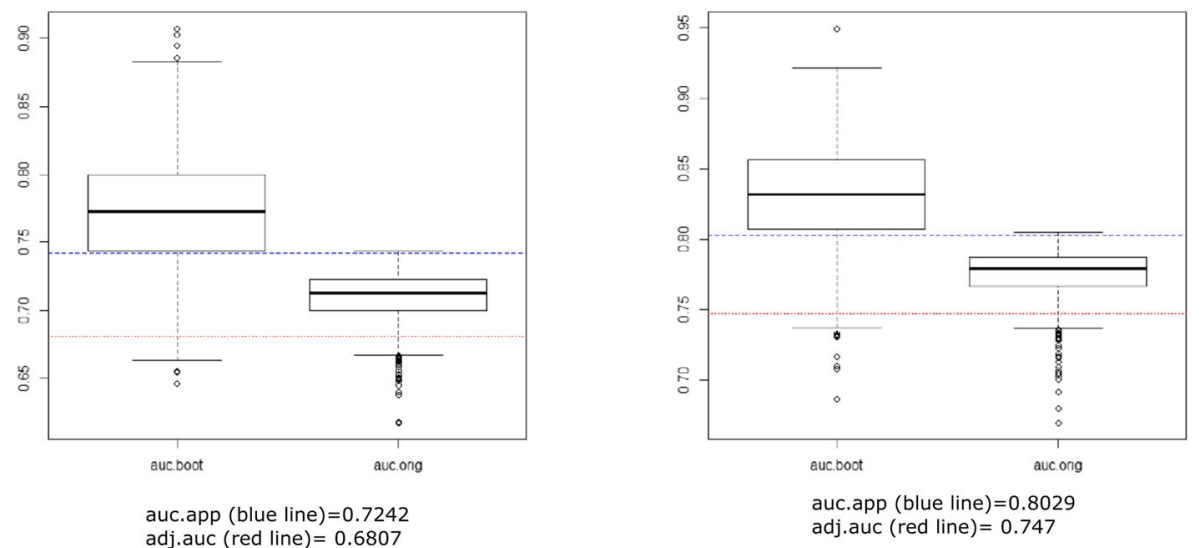

Fig. 4 Correction for optimism of AUC for both core and extended models. Auc.boot is the distribution of the AUC value in the bootstrap sample, which represents "an estimation of the apparent performance." "auc.orig" is the distribution of the AUC value deriving from the model fitted to the bootstrap samples and evaluated on the original sample, which represents the model performance on independent data. At the bottom of the chart, the apparent AUC (i.e., the value deriving from the model fitted to the original dataset) and the AUC adjusted for optimism are reported on the box plot respectively with the blue line and red line [25]

for detecting brain ischemia, may have also played a role. Not least, the centralized revision of all brain CTs increased the detection of PTCI, as 10 more PTCI cases were identified compared to diagnoses made locally by participating centers. This happened without sacrificing specificity because 7 patients initially classified as PTCI were subsequently classified as non-PTCI after centralized revision.

Our study has strengths and limitations. Strengths include the prospective multicenter nature of the study with central readings of all brain CT scans, as well as rigorous statistical methodology.

The identification of PTCI in ambiguous cases was greatly aided by the centralized evaluation of the neuroimaging data, allowing the assessment of the entire neuroradiological history of each patient, with immediate comparison of any questionable finding in a given $\mathrm{CT}$ scan to several previous and following exams, thus making the final diagnosis of cerebral infarction much more reliable.

The main limitation is the absence of detailed neurological and neuropsychological assessments of patients at long-term follow-up, limiting our understanding of the relative contribution of the primary traumatic and secondary ischemic brain damage to the persisting disability. Another limitation is that we did not assess the causes of mortality, and hence, we cannot exclude that life-sustaining therapies were withdrawn in patients with PTCI, leading to a self-fulfilling prophecy bias of the outcome prediction model. Moreover, results became available to the participating centers only after the entire brain CT data set of each patient was transferred to the coordinating center where the diagnosis of PTCI was definitely determined. 
Table 4 Adjusted logistic regression for dichotomized GOS (favorable outcome: GOS = 4 and 5, and unfavorable outcome: GOS < 4) for core model and extended model with and without the addition of PTCl. AUC and AIC for each model are represented along with the ANOVA comparison between the model with and without the addition of PTCI. We used Nahelkerke's Pseudo $R^{2}$ for consistency with the original IMPACT study

\begin{tabular}{|c|c|c|c|c|c|c|}
\hline & Core & & & Exten & & \\
\hline & $\mathrm{OR}$ & 95\% C.I. & $p$ & $\mathrm{OR}$ & 95 C.I. & $p$ \\
\hline Age & 1.03 & $1.01-1.06$ & 0.002 & 1.01 & $1.01-1.07$ & 0.002 \\
\hline GCSm & 0.34 & $0.14-0.77$ & 0.012 & 0.33 & $1.27-7.14$ & 0.015 \\
\hline Pupils & 2.76 & $1.08-7.35$ & 0.035 & 1.79 & $0.64-5.05$ & 0.263 \\
\hline PTCl & & & & 4.52 & $1.88-11.39$ & $<0.001$ \\
\hline AUC & 0.73 & $-0.82)$ & & 0.79 & $-0.83)$ & \\
\hline AUC comparison & Mode & $s$ better than & without & & & \\
\hline Nagelkerke's R ${ }^{2}$ & 0.21 & & & 0.33 & & \\
\hline Age & 1.03 & $1.01-1.06$ & 0.003 & 1.03 & $1.01-1.06$ & 0.004 \\
\hline GCSm & 0.29 & $0.12-0.07$ & 0.007 & 0.27 & $0.10-0.67$ & 0.007 \\
\hline Pupils & 3.11 & $1.14-8.90$ & 0.027 & 2.09 & $0.68-6.48$ & 0.190 \\
\hline Hypotension & 2.24 & $0.73-7.12$ & 0.160 & 3.29 & $0.99-11.60$ & 0.055 \\
\hline Hypoxia & 1.58 & $0.41-6.01$ & 0.495 & 2.00 & $0.43-11.63$ & 0.37 \\
\hline SAH & 1.23 & $0.49-3.19$ & 0.652 & 1.30 & $0.46-3.80$ & 0.62 \\
\hline EDH & 0.93 & $0.28-0.41$ & 0.882 & 7.96 & $0.26-2.28$ & 0.68 \\
\hline Marshall CT score & - & - & 0.991 & - & - & 0.99 \\
\hline $\mathrm{PTCl}$ & & & & 6.84 & $2.58-19.7$ & $<0.001$ \\
\hline AUC & 0.74 & & & 0.82 & & \\
\hline Nagelkerke's $\mathrm{R}^{2}$ & 0.26 & & & 0.39 & & \\
\hline AUC comparison & Mode & $s$ better than & without & 008 & & \\
\hline
\end{tabular}

GCSm Glasgow Coma Scale motor score, $O R$ proportional odds ratio, C.I. confidence interval, SAH traumatic subarachnoid hemorrhage, EDH epidural hematoma. Hypotension and hypoxia refer to both pre-hospital and intra-hospital periods

Our study is the first multicenter study demonstrating that a cerebral infarction, indicating posttraumatic brain damage, is an independent predictor of long-term disability when added to validated prediction models. This is in line with the recommendation of the Lancet Neurology Commission that prognostic models for TBI patients should include dynamic predictors that develop during the disease course [1]. Future research should externally validate this finding in larger studies with adequate power and accurate neurological and neuropsychological assessments of patients at longerterm follow-up. This would assess its generalizability and recommend the inclusion of PTCI in the list of measurable, clinically relevant variables that improve prognostication and contribute to a comprehensive definition of the diversity of post-TBI disability and the needs for personalized rehabilitation. The global burden of TBI has continuously increased in the last 25 years [30], and the prevention of residual disability is a major concern. Future studies should prioritize at riskpatients identification, along with effective prevention strategies to be deployed before the cerebral infarction is fully established.

\section{Conclusions}

The findings provide evidence that PTCI is a common complication in patients suffering from a moderate or severe TBI and is an independent risk factor for longterm disability. The addition of PTCI to the IMPACT core and extended predictive models significantly increased their performance in predicting the GOS.

\section{Abbreviations}

PTCI: Posttraumatic cerebral infarction; TBI: Traumatic brain injury:

ICUs: Intensive care units; GOS: Glasgow Outcome Scale;

IMPACT: International Mission for Prognosis and Clinical Trial design in TBI; GCSm: Glasgow Coma Scale motor score; CT: Computed tomography; DICOM: Digital Imaging and Communications in Medicine;

SAH: Subarachnoid hemorrhage; EDH: Epidural hematoma; ACA: Anterior cerebral artery; MCA: Middle cerebral artery; PCA: Posterior cerebral artery; LSAs: Lenticulostriate arteries; TPAs: Thalamo-perforating arteries; BA: Basilar artery; AICA: Anterior-inferior cerebellar artery; SCA: Superior cerebellar artery; PICA: Posterior-inferior cerebellar artery; LOS: Length of stay; AUC: Receiver operating characteristic curve; ICP: Intracranial pressure

\section{Acknowledgements}

None

Authors' contributions

NL was the lead intensivist, had full access to all study data, and take responsibility for the study integrity and the accuracy of data analysis. SP performed the statistical analyses with supervision from Cosetta Minelli, did 
the literature search, contributed to data interpretation, and wrote the final version of the manuscript. LP and MF did the radiological analyses with the central revision of all brain CTs, led the adjudication of neuroimaging, and contributed to the analysis and interpretation of the data. NF was responsible for data acquisition, coordinated the information technology and database, and contributed to statistical analyses, data interpretation, and manuscript writing. DT contributed to data collection, coordination of individual sites, statistical analyses, and data interpretation. MB, AB, LC, TC, Carlo CAS, SC, DC, GC, SG, AG, $P G, O M, P M, R M, G N, M P, M E P, I P, D K R$, and MS were responsible for the conduct of the study, data acquisition, and interpretation. MF contributed to the interpretation of clinical and neuroradiological data. RS contributed to the study concept, design, and conduct, and interpretation of clinical and neuroradiological data. CM supervised the statistical analyses and contributed to data interpretation and manuscript writing. RG did the radiological analyses with the central revision of all brain CTs and led the adjudication of neuroimaging. FAR conceived and designed the study, contributed to data analysis and interpretation, and wrote the final version of the manuscript. NF and SP equally contributed to this work. FAR and NL equally contributed to this work. All authors critically revised the manuscript for important intellectual content and approved the final manuscript.

\section{Funding}

The present study was self-funded.

\section{Availability of data and materials}

Dataset of the present paper and R Code are available at Piva, Simone (2019), "Neurology-INCEPT Study Dataset", Mendeley Data, https://data. mendeley.com/datasets/publish-confirmation/62thth3yyd/1

\section{Ethics approval and consent to participate}

The study was conducted in accordance with the Declaration of Helsinki and was approved by the local ethics committees of each participating center. Patients' informed consent was waived due to the lack of definition of a legal representative of temporarily incapacitated adult patients in the Italian legislation. The informed consent was obtained from the surviving patients as soon as they regained their mental competency. Family members received detailed information on the study scope and protocol.

\section{Consent for publication}

All the authors consent for publication of the present study. Informed consent was obtained from patients for the publication of their individual data.

\section{Competing interests}

The authors declare that they have no competing interests.

\section{Author details}

${ }^{1}$ Department of Medical and Surgical Specialties, Radiological Sciences and Public Health, University of Brescia, Brescia, Italy. ${ }^{2}$ Department of Anesthesia, Intensive Care and Emergency, ASST Spedali Civili University Hospital, Piazzale Ospedali Civili 1, 25121 Brescia, Italy. ${ }^{3}$ Department of Molecular and Translational Medicine, University of Brescia, Brescia, Italy. ${ }^{4}$ Department of Radiology, Neuroradiology Unit, ASST Spedali Civili University Hospital, Brescia, Italy. ${ }^{5}$ Anesthesia and Intensive Care Unit, AOU Città della Salute e della Scienza, Presidio CTO, Turin, Italy. ${ }^{6}$ Department of Anesthesia and Critical Care Medicine, IRCCS Policlinico San Matteo Pavia, Pavia, Italy. ${ }^{7}$ Department of Anestesiology and Intensive Care Medicine, S. Giovanni Bosco Hospital, ASLTO2, Turin, Italy. ${ }^{8}$ Department of Neuroradiology, Ospedale Maggiore, University of Parma, Parma, Italy. ${ }^{9}$ School of Medicine and Surgery, University of Milan-Bicocca, Milan, Italy. ${ }^{10}$ Department of Anesthesia and Critical Care Medicine, Unit of Neurointensive Care Medicine, ASST-Monza, Monza, Italy. ${ }^{11}$ Division of Neurosurgery, Department of Medical and Surgical Specialties, Radiological Sciences and Public Health, University of Brescia, Brescia, Italy. ${ }^{12}$ Department of Anesthesia and Critical Care Medicine, Azienda Ospedaliera Universitaria Pisana, Pisa, Italy. ${ }^{13}$ Department of Anesthesia and Intensive Care, Fondazione Poliambulanza Hospital, Brescia, Italy. ${ }^{14}$ Department of Anesthesia and Critical Care Medicine, Papa Giovanni XXIII Hospital, ASST Bergamo, Bergamo, Italy. ${ }^{15}$ Department of Neuroradiology, Papa Giovanni XXIII Hospital, ASST Bergamo, Bergamo, Italy. ${ }^{16}$ Department of Anesthesiology, Critical Care and Pain Medicine, Maggiore University Hospital, Parma, Italy. ${ }^{17}$ Department of Radiology, Section of
Neuroradiology, ASST-Monza, Monza, Italy. ${ }^{18}$ Department of Radiology, S. Giovanni Bosco Hospital, ASLTO2, Turin, Italy. ${ }^{19}$ Department of Diagnosis and Imaging, Neuroradiology, Azienda Ospedaliera Universitaria Pisana, Pisa, Italy. ${ }^{20}$ Department of Neuroscience, Legnano Hospital, Milan, Italy. ${ }^{21}$ The National Heart and Lung Institute (NHLI), Imperial College London, London, UK.

Received: 28 September 2019 Accepted: 16 January 2020

Published online: 03 February 2020

\section{References}

1. Maas AIR, Menon DK, Adelson PD, Andelic N, Bell MJ, Belli A, et al. Traumatic brain injury: integrated approaches to improve prevention, clinical care, and research. Lancet Neurol. 2017;16(12):987-1048.

2. Lingsma HF, Roozenbeek B, Steyerberg EW, Murray GD, Maas Al. Early prognosis in traumatic brain injury: from prophecies to predictions. Lancet Neurol. 2010;9(5):543-54.

3. Maas AIR, Murray GD, Roozenbeek B, Lingsma HF, Butcher I, McHugh GS, et al. Advancing care for traumatic brain injury: findings from the IMPACT studies and perspectives on future research. Lancet Neurol. 2013;12(12): 1200-10.

4. Marino R, Gasparotti R, Pinelli L, Manzoni D, Gritti P, Mardighian D, et al. Posttraumatic cerebral infarction in patients with moderate or severe head trauma. Neurology. 2006;67(7):1165-71.

5. Chen H, Xue L-X, Guo Y, Chen S-W, Wang G, Cao H-L, et al. The influence of hemocoagulation disorders on the development of posttraumatic cerebral infarction and outcome in patients with moderate or severe head trauma. Biomed Res Int. 2013;2013:1-9.

6. Mirvis SE, Wolf AL, Numaguchi Y, Corradino G, Joslyn JN. Posttraumatic cerebral infarction diagnosed by CT: prevalence, origin, and outcome. AJR Am J Roentgenol. 1990;154(6):1293-8.

7. Tawil I, Stein DM, Mirvis SE, Scalea TM. Posttraumatic cerebral infarction: incidence, outcome, and risk factors. J Trauma Inj Infect Crit Care. 2008;64(4): 849-53.

8. Liu S, Wan X, Wang S, Huang L, Zhu M, Zhang S, et al. Posttraumatic cerebral infarction in severe traumatic brain injury: characteristics, risk factors and potential mechanisms. Acta Neurochir (Wien). 2015;157(10):1697-704.

9. Bae D-H, Choi K-S, Yi H-J, Chun H-J, Ko Y, Bak KH. Cerebral infarction after traumatic brain injury: incidence and risk factors. Korean J Neurotrauma. 2014;10(2):35-40.

10. Bratton SL, Chestnut RM, Ghajar J, McConnell Hammond FF, Harris OA, Hartl R, et al. Guidelines for the management of severe traumatic brain injury. VII. Intracranial pressure monitoring technology. J Neurotrauma. 2007;24(Suppl 1): S45-54.

11. Zamperetti N, Latronico N. Clinical research in critically ill patients: the situation in Italy. Intensive Care Med. 2008;34(7):1330-2.

12. von Elm E, Altman DG, Egger M, Pocock SJ, Gøtzsche PC, Vandenbroucke JP. STROBE Initiative. The Strengthening the Reporting of Observational Studies in Epidemiology (STROBE) statement: guidelines for reporting observational studies. J Clin Epidemiol. 2008;61(4):344-9.

13. Marshall LF, Becker DP, Bowers SA, Cayard C, Eisenberg H, Gross CR, et al. The National Traumatic Coma Data Bank. Part 1: Design, purpose, goals, and results. J Neurosurg. 1983;59(2):276-84.

14. Marshall LF, Toole BM, Bowers SA. The National Traumatic Coma Data Bank. Part 2: Patients who talk and deteriorate: implications for treatment. J Neurosurg. 1983;59(2):285-8.

15. Servadei F, Murray GD, Penny K, Teasdale GM, Dearden M, lannotti F, et al. The value of the «worst» computed tomographic scan in clinical studies of moderate and severe head injury. Eur Brain Injury Consortium Neurosurgery. 2000;46(1):70-5 discussion 75-7.

16. Brown CVR, Zada G, Salim A, Inaba K, Kasotakis G, Hadjizacharia P, et al. Indications for routine repeat head computed tomography (CT) stratified by severity of traumatic brain injury. J Trauma - Inj Infect Crit Care. 2007;62(6): 1339-44.

17. National Institute for Health and Clinical. Head injury: triage, assessment, investigation and early management of head injury in infants, children and adults. NICE clinical guideline No 56. 2007.

18. Tatu L, Moulin T, Bogousslavsky J, Duvernoy H. Arterial territories of human brain: brainstem and cerebellum. Neurology. 1996;47(5):1125-35.

19. Tatu L, Moulin T, Bogousslavsky J, Duvernoy H. Arterial territories of the human brain: cerebral hemispheres. Neurology. 1998;50(6):1699-708. 
20. Tatu L, Moulin T, Vuillier F, Bogousslavsky J. Arterial territories of the human brain. Front Neurol Neurosci. 2012;30:99-110.

21. Jennett B, Bond M. Assessment of outcome after severe brain damage. Lancet Lond Engl. 1975;1(7905):480-4.

22. Feinstein AR. Clinical epidemiology: the architecture of clinical research. USA: W. B. Saunders Compan, curatore. Philadelphia; 1985.

23. McCullagh P. Regression models for ordinal data. J R Stat Soc Ser B. 1980;42:109-42.

24. Steyerberg EW, Harrell FE, Borsboom GJ, Ejijkemans MJ, Vergouwe Y, Habbema JD. Internal validation of predictive models: efficiency of some procedures for logistic regression analysis. J Clin Epidemiol. 2001;54(8):774-81.

25. Alberti G. «auc.adjust»: R function for optimism-adjusted AUC (internal validation). 2014

26. Pencina MJ, D'Agostino RB, Pencina KM, Janssens ACJW, Greenland P. Interpreting incremental value of markers added to risk prediction models. Am J Epidemiol. 2012;176(6):473-81.

27. Rodgers $\mathrm{H}$, Thomson R. Functional status and long term outcome of stroke. BMJ. 2008;336(7640):337-8

28. Kelly-Hayes M, Beiser A, Kase CS, Scaramucci A, D'Agostino RB, Wolf PA. The influence of gender and age on disability following ischemic stroke: the Framingham study. J Stroke Cerebrovasc Dis Off J Natl Stroke Assoc. 2003;12(3):119-26.

29. Holloway RG, Benesch CG, Burgin WS, Zentner JB. Prognosis and decision making in severe stroke. JAMA. 2005;294(6):725-33.

30. James SL, Theadom A, Ellenbogen RG, Bannick MS, Montjoy-Venning W, Lucchesi $L R$, et al. Global, regional, and national burden of traumatic brain injury and spinal cord injury, 1990-2016: a systematic analysis for the global burden of disease study 2016. Lancet Neurol. 2019;18(1):56-87.

\section{Publisher's Note}

Springer Nature remains neutral with regard to jurisdictional claims in published maps and institutional affiliations.

Ready to submit your research? Choose BMC and benefit from:

- fast, convenient online submission

- thorough peer review by experienced researchers in your field

- rapid publication on acceptance

- support for research data, including large and complex data types

- gold Open Access which fosters wider collaboration and increased citations

- maximum visibility for your research: over $100 \mathrm{M}$ website views per year

At $\mathrm{BMC}$, research is always in progress.

Learn more biomedcentral.com/submissions 